\title{
Prostatic Monocyte Chemotactic Protein-1 (MCP-1): A Novel Potential Biomarker for Symptomatic Benign Prostatic Hyperplasia
}

\author{
Kazutoshi Fujita, ${ }^{1,2}$ J. Kellogg Parsons, ${ }^{3}$ Charles M. Ewing, ${ }^{1}$ George J Netto ${ }^{1}$, William B. Isaacs ${ }^{1}$ and \\ Christian P. Pavlovich ${ }^{1}$ \\ ${ }^{1}$ The Brady Urological Institute, Johns Hopkins Medical Institutions, Baltimore, MD, USA \\ ${ }^{2}$ Department of Urology, Osaka University Graduate School of Medicine, Suita, Japan \\ ${ }^{3}$ Division of Urologic Oncology, Moores Comprehensive Cancer Center, University of California, San Diego and \\ Division of Urology, San Diego Veterans Affairs Medical Center, La Jolla, CA, USA \\ Email: cpavlov2@jhmi.edu
}

\begin{abstract}
Monocyte chemotactic protein-1 (MCP-1) is differentially expressed in benign prostatic hyperplasia $(\mathrm{BPH})$ and is a potential clinical biomarker for $\mathrm{BPH}$. We collected urine post-digital rectal examination (DRE) from 48 men who did not have a diagnosis of prostate cancer. We measured MCP-1 levels by enzyme-linked immunosorbent assay (ELISA) and compared them with prostate weight and lower urinary tract symptoms (LUTS) as measured by the International Prostate Symptom Score (I-PSS) utilizing correlation and regression analyses. In post-DRE urine, higher MCP-1 levels were associated with increased I-PSS but not with prostate volume. MCP-1 levels in men with prostate enlargement were significantly higher than those in men without enlargement. In multivariable regression adjusting for age, prostate volume, and PSA,higher urinary MCP-1 was associated with significantly higher I-PSS. Since MCP-1 is preferentially expressed in BPH tissue, these data suggest that MCP-1 may be a novel biomarker for clinically significant BPH.
\end{abstract}

Keywords: MCP-1, LUTS, urine marker, benign prostatic hyperplasia

\section{Introduction}

Benign prostate hyperplasia $(\mathrm{BPH})$ is a common disease affecting older men caused by unregulated prostatic stromal and epithelial growth resulting in prostate enlargement, bladder outlet obstruction, and lower urinary tract symptoms (LUTS) [1]. Despite intensive researches over the last several decades, the molecular mechanisms underlying prostatic enlargement and symptomatic bladder outlet obstruction remain obscure, and specific clinical markers for symptomatic BPH have yet to be developed.

We previously used a human cytokine array to search for cytokines in expressed prostatic secretions (EPS) from radical prostatectomy specimens that may be associated with prostate enlargement, and found that monocyte/macrophage chemoattractant protein-1 (MCP-1, CCL2) in EPS was associated with prostate size [2]. MCP-1 is a member of the CC chemokine superfamily and plays a critical role in the recruitment and activation of monocytes during inflammation [3]. MCP-1 is secreted from prostatic stromal cells and stimulates the proliferation of prostatic epithelial cells in vitro. Immunohistochemical studies indicated that MCP-1 was upregulated in benign prostatic hyperplasia (BPH) lesions, and MCP-1 levels in EPS correlated with mRNA levels of the macrophage marker CD68 [4]. Based on these data, we hypothesize that MCP-1 levels in urine after DRE, which is enriched in prostatic contents, could serve as a specific clinical marker for symptomatic BPH. Therefore, we evaluated associations of MCP-1 levels in urine collected after DRE with prostate volume and LUTS by enzyme-linked immunosorbent assay (ELISA).

\section{$2 \quad$ Materials and Methods}




\subsection{Sample Collection}

Urine samples were collected in the urology clinic from men presenting for prostate biopsy (for abnormal PSA and/or DRE findings). None of these men had a prior history of prostate cancer, and they had varying levels of LUTS. Approval was obtained from our Institutional Review Board before initiating the study and all patients provided written informed consent prior to providing urine samples. Initial voided urine specimens (10 to $100 \mathrm{ml}$ ) were prospectively collected from 48 men with a mean age of 62.2 years immediately following an attentive DRE during a single office visit. Voided urine specimens were centrifuged for 10min at $1000 \mathrm{~g}$ to remove sediments. Urine supernatants were kept at $-80 \mathrm{oC}$ until analysis. All patients subsequently underwent twelve-core transrectal ultrasound-guided prostate needle biopsy that was negative for malignancy. Prostate volume was calculated transrectally with the ultrasound probe using the formula for an ellipsoid. LUTS were assessed with the International Prostate Symptom Score (I-PSS) prior to the DRE and subsequent biopsy.

\subsection{ELISA}

MCP-1 levels were measured by ELISA with a human CCL2 (MCP-1) ELISA Ready-SET-GO kit (eBioscience, San Diego, CA). MCP- 1 measurements were performed as per the manufacturer's recommendations. Urine samples were assayed at 16 -fold dilutions.

\subsection{Statistical Methods}

We defined "mild", "moderate" and "severe" LUTS as International Prostate Symptom Score (I-PSS) = $0-7,8-19$, and $20-35$ respectively. We defined prostate enlargement categorically as prostate volume $\geqslant 40$ cc. We used Spearman correlation analysis to evaluate associations of MCP-1 with I-PSS and prostate volume. We compared MCP-1 levels of the group categorized by I-PSS or prostate volume by Kruskal-Wallis test and Mann-Whitney test, respectively. We examined MCP-1 levels in urine with prostate volume and I-PSS utilizing regression modeling adjusted for age [5], serum PSA, and prostate volume. Statistical significance was defined as a $\mathrm{p}$ value $<0.05$.

\section{$3 \quad$ Results}

\subsection{Demographics}

Median serum PSA level was $5.25 \mathrm{mg} / \mathrm{ml}$ (range $1.2-14.5$ ) and median prostate volume was $38.5 \mathrm{ml}$ (range 20 - 102). Median I-PSS was 9 (range 0- 33). 21 men (43.7\%) were using alpha1- blocker medication.

\subsection{MCP-1 in Post-DRE Urine}

MCP-1 in post-DRE urine was detectable in 25 men (52.0\%) by quantitative ELISA. The median MCP-1 level in post-massage urine was $3.99 \mathrm{pg} / \mathrm{ml}$ (range $0.0-431.5)$. Spearman correlation analysis showed that MCP-1 was positively associated with I-PSS $(\mathrm{r}=0.376, \mathrm{P}=0.022)$ (Figure 1a) but not with prostate volume $(\mathrm{r}=0.190, \mathrm{P}=0.194)$ (Figure $1 \mathrm{~b})$. MCP-1 was also positively associated with serum PSA $(\mathrm{r}=$ $0.362, \mathrm{P}=0.016$ ). MCP-1 levels in post-DRE urine from men with mild symptoms (I-PSS: $0-7$ ) were significantly lower (median $0.0 \mathrm{pg} / \mathrm{ml}$, range $0.0-102.9$ ) than those from men with moderate symptoms (I-PSS: 8 - 19) (median $71.2 \mathrm{pg} / \mathrm{ml}$, range $0.0-431.5)$ and men with severe symptoms (I-PSS: $20-35$ ) (median $6.3 \mathrm{pg} / \mathrm{ml}$, range $0.0-332.0)(\mathrm{P}<0.01)$ (Figure $2 \mathrm{a}$ ). MCP-1 in post-DRE urine was detectable in $29.4 \%$ of men with mild symptoms compared to $70.0 \%$ of men with moderate or severe symptoms $(\mathrm{P}=$ 0.01). MCP-1 levels in post-DRE urine from men with prostate enlargement (prostate volume $>40 \mathrm{cc}$ ) were significantly higher (median $21.0 \mathrm{pg} / \mathrm{ml}$, range $0.0-431.5)$ than those from men without prostate enlargement (median $0.0 \mathrm{pg} / \mathrm{ml}$, range $0.0-169.6)(\mathrm{P}=0.02)$ (Figure $2 \mathrm{~b}$ ). 
a

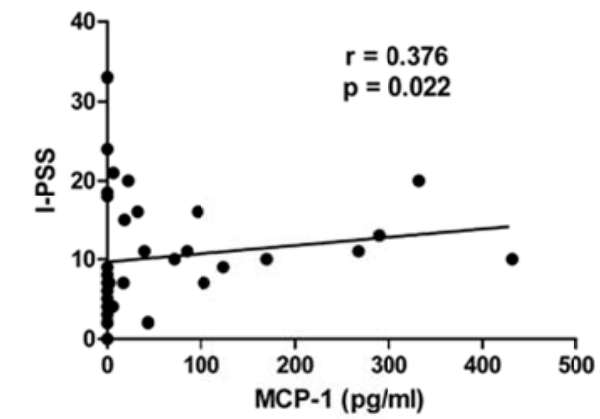

b

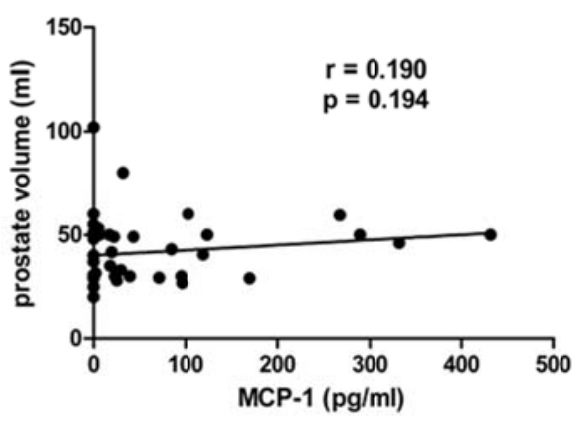

Figure 1. Correlation of I-PSS (a) and prostate volume (b) with urinary MCP-1 levels by Spearman correlation coefficient. (r: Spearman r)

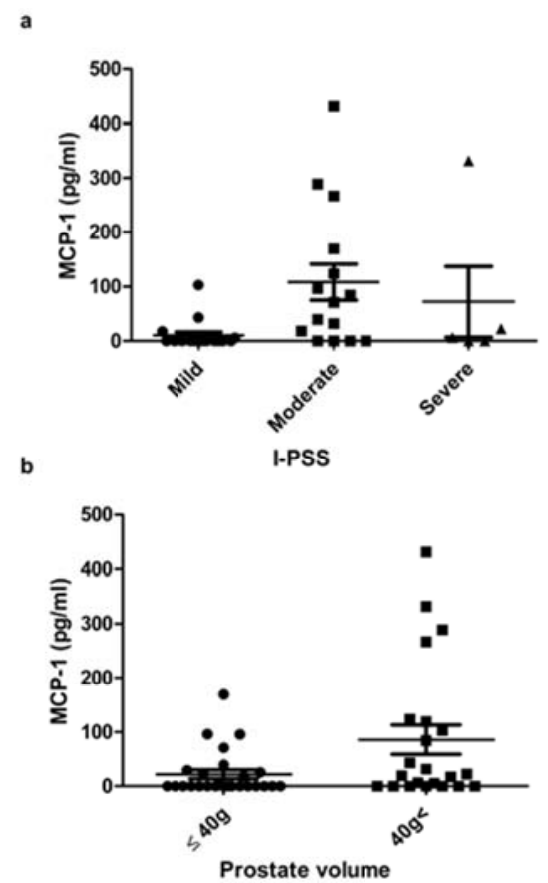

Figure 2. MCP-1 levels in post-DRE urine. (a) MCP-1 levels stratified by I-PSS. (Mild: I-PSS 0 - 7, Moderate: I-PSS $8-16$, Severe: I-PSS 17 - 25). MCP-1 levels in urine from men with mild symptoms were significantly lower than those from men with moderate symptoms (I-PSS: 8 - 19) and men with severe symptoms ( $p<0.01$ ). (b) MCP-1 levels stratified by prostate volume. MCP-1 levels in urine from men with prostate enlargement were significantly higher than those from men without prostate enlargement $(\mathrm{p}=0.02)$. 


\subsection{Multivariable Model of MCP-1 and I-PSS}

In multivariable regression adjusting for age, PSA, and prostate volume, higher urinary MCP-1 was associated with significantly higher I-PSS $(\mathrm{P}=0.05)$, but not with prostate volume $(\mathrm{P}=0.44)$.

\section{Discussion}

In this study, we observed that increased MCP-1 levels in urine were associated with increasing LUTS, independent of age and prostate volume. Since MCP-1 is preferentially expressed in BPH lesions, these data suggest that MCP-1 may be a novel biomarker for LUTS secondary to BPH, and that prostate-generated inflammatory mediators may substantially contribute to LUTS pathogenesis in men with BPH.

We have previously demonstrated that MCP-1 is highly expressed in histological BPH [2]. Prostatic stromal cells secrete MCP-1, and MCP-1 stimulates the proliferation of prostatic epithelial cells in vitro. MCP-1 mRNA levels are significantly lower in prostate cancer specimens than in normal prostate specimens [6]. In prostate cancer, the number of MCP-1 mRNA expressing cells is significantly less than in BPH4; MCP-1 mRNA expression is located in fibromuscular stroma and in basal cells of the prostate, whereas it is absent in the secretory cells of normal glands and prostate cancer. However, MCP-1 is also associated with prostate cancer development; it is a potent chemoattractant of prostate cancer cells [7], and targeting MCP-1 with neutralizing antibodies induces prostate cancer regression in mice [8].

Urine is likely to become an increasingly powerful source of prostate-specific biomarkers [9-12]. Urine after DRE contains various markers originating from the prostate, including cytokines10, RNAs [13], DNAs [14], and prostate cells [11]. MCP-1 was assayed in the urine after DRE in order to sample the prostatic microenvironment most directly. DRE exerts pressure on much of the prostate, and at least in theory allows for a sampling of secretions from the entire gland, unlike a prostate biopsy. It is known that the initial urine obtained after DRE is enriched in prostatic proteins, and we have previously demonstrated that MCP-1 is increased in fluid obtained from radical prostatectomy specimens of large volume2, suggesting that MCP-1 levels in body fluids (prostatic secretions and urine) may potentially serve as markers for BPH, chronic prostatitis/chronic pelvic pain syndrome (CPPS), and LUTS. Prostatic inflammation also causes PSA elevation, and urinary MCP-1 levels may help distinguish benign conditions such as nonspecific inflammation from prostate cancer.

Recently, MCP-1 and another monocyte/macrophage chemoattractant molecule, macrophage inflammatory protein-1a (MIP-1a), have been proposed as possible biomarkers for CPPS, with intriguing data to support this [15]. In a careful study of EPS obtained in the clinic from a cohort of young male controls and CPPS patients, as well as from older men with BPH, elevated MCP-1 and MIP-1a levels correlated with the CPPS diagnosis, and MCP-1 and MIP-1a levels were also slightly elevated in BPH, although neither prostate volume nor I-PSS score were described.

Correlation analysis showed that there was no linear association of MCP-1 with prostate volume, while MCP-1 levels were significantly higher in the group with prostate enlargement (prostate volume $>40 \mathrm{cc}$ ) than without enlargement. MCP-1 in urine is also produced from kidney and bladder as well as prostate. These varieties of MCP-1 sources might have caused this discrepancy. Since MCP-1 stimulated the proliferation of prostatic epithelial cells in vitro, MCP-1 levels above a certain threshold might cause the prostatic enlargement.

In this study, MCP-1 levels were lower in urine from patients with severe symptoms than with moderate symptoms. Desireddi et al. reported that MCP-1 levels were lower in urine from patients with inflammatory chronic prostatitis/CPPS (IIIA) than from patients with noninflammatory chronic prostatitis/CPPS (IIIB) [14]. MCP-1 levels in urine were also higher in the women with overactive bladder than the normal women [16]. This study had only five cases with severe symptoms, but one possible explanation might be that inflammatory chronic prostatitis caused the severe symptoms, leading to lower MCP-1 levels in urine. Tyagi et al. studied the association of urinary levels of MCP-1, CXCL-1, CXCL-8, CXCL-10, CCl3 and sIL-1ra with BPH/LUTS and urine levels of CXCL-8, CXCL-10, and sIL-1ra were associated with BPH/LUTS [17]. Tyagi et al. used the urine without massage, which might cause the difference from our results. 
Our study has several limitations. All men included in this study underwent prostate biopsy for abnormal DRE or elevated PSA. Inflammation might have caused PSA elevation and skewed the MCP-1 levels in some of our patients. Men with normal PSA should be tested for MCP-1 levels in urine associated with LUTS. MCP-1 levels in urine should be measured before and after DRE in individuals to confirm its prostatic origin. DRE might be necessary to measure the MCP-1 from prostate. In this study, only 48 men were included, and further larger scale study will be needed.

\section{Conclusions}

Increased MCP-1 in urine after DRE is associated with increased LUTS. There is no biologic marker for $\mathrm{BPH}$ in clinical use to date; therefore, a molecule such as MCP-1 presents a novel, potentially important urinary marker for the presence and/or development of symptomatic BPH.

\section{Conflict of Interest:}

Funding Sources: NIDDK 1K23DK071262, DOD W81XWH-05-1-0167

Dr Fujita, Dr Parsons and Dr Pavlovich filed the U.S. patent application (PCT/US2010/030736, METHODS FOR THE DIAGNOSIS AND TREATMENT OF BENIGN PROSTATIC HYPERPLASIA). J. Kellogg Parsons is a consultant/speaker for AMS. Mr Ewing and Dr Isaacs declare no potential conflict of interest.

\section{References}

1. J. T. Wei, E. Calhoun, S. J. Jacobsen, "Urologic diseases in America project: benign prostatic hyperplasia." Journal of Urology, vol. 173, no. 4, pp. 1256-1261, 2005.

2. K. Fujita, C. M. Ewing, R.H. Getzenberg, J. K. Parsons, W. B. Isaacs, C. P. Pavlovich, "Monocyte chemotactic protein-1 (MCP-1/CCL2) is associated with prostatic growth dysregulation and benign prostatic hyperplasia", Prostate, vol. 70, no. 5, pp. 473-481, 2010; 70.

3. N. Mukaida, A. Harada, K. Matsushima, "Interleukin-8 (IL-8) and monocyte chemotactic and activating factor (MCAF/MCP-1), chemokines essentially involved in inflammatory and immune reactions". Cytokine \& Growth Factor Reviews, vol. 9, no. 1, pp. 9-23, 1998.

4. L. Mazzucchelli, P. Loetscher, A. Kappeler, M. Uguccioni, M. Baggiolini, J. A. Laissue et al. "Monocyte chemoattractant protein-1 gene expression in prostatic hyperplasia and prostate adenocarcinoma". American Journal of Pathology, vol. 149, no. 2, pp. 501-509, 1996.

5. J. K. Parsons, J. Bergstrom, J. Silberstein, E. Barrett-Connor. Prevalence and characteristics of lower urinary tract symptoms in men aged $>$ or $=80$ years. Urology, vol. 72, no. 2, pp. 318-321, 2008.

6. A. Chetcuti, S. Margan, S. Mann, P. Russell, D. Handelsman, J. Rogers et al. "Identification of differentially expressed genes in organ-confined prostate cancer by gene expression array". Prostate, vol. 47, no. 2, pp. 132-140, 2001.

7. R. D. Loberg, L. L. Day, J. Harwood, C. Ying, L. N. St John, R. Giles et al. "CCL2 is a potent regulator of prostate cancer cell migration and proliferation". Neoplasia, vol. 8, no. 7, pp. 578-586, 2006.

8. R. D. Loberg, C. Ying, M. Craig, L. L. Day, E. Sargent, C. Neeley et al. "Targeting CCL2 with systemic delivery of neutralizing antibodies induces prostate cancer tumor regression in vivo." Cancer Research, vol. 67, no. 19, pp. 9417-9424, 2007.

9. B. Laxman, D. S. Morris, J. Yu, J. Siddiqui, J. Cao, R. Mehra et al." A first-generation multiplex biomarker analysis of urine for the early detection of prostate cancer". Cancer Research, vol. 68, no. 3, pp. 645-649, 2008.

10.K. Fujita, C. M. Ewing, D. Y. Chan, L. A. Mangold, A. W. Partin, W. B. Isaacs et al. "Endoglin (CD105) as a urinary and serum marker of prostate cancer". International Journal of Cancer, vol. 124, no. 3, pp. 664-669, 2009.

11.K. Fujita, C. P. Pavlovich, G. J. Netto, Y. Konishi, W. B. Isaacs, S. Ali et al. "Specific detection of prostate cancer cells in urine by multiplex immunofluorescence cytology". Human Pathology, vol. 40, no.7, pp. 924-933, 2009.

12.K. Fujita, T. Hayashi, K. Matsuzaki, W. Nakata, M. Masuda, A. Kawashima et al. "Decreased fucosylated PSA as a urinary marker for high Gleason score prostate cancer". Oncotarget.10987, 2016. 
13.J. J. Tosoian, S. Loeb, A. Kettermann, P. Landis, D. J. Elliot, J. I. Epstein et al. "Accuracy of PCA3 measurement in predicting short-term biopsy progression in an active surveillance program". Journal of Urology, vol. 183, no.2, pp. 534-538, 2010.

14.J. Baden, G. Green, J. Painter, K. Curtin, J. Markiewicz, J. Jones et al. "Multicenter evaluation of an investigational prostate cancer methylation assay". Journal of Urology, vol. 182, no.3, pp. 1186-1193, 2009.

15.N. V. Desireddi, P. L. Campbell, J. A. Stern, R. Sobkoviak, S. Chuai, S. Shahrara et al. "Monocyte chemoattractant protein-1 and macrophage inflammatory protein-1alpha as possible biomarkers for the chronic pelvic pain syndrome". Journal of Urology, vol. 179, no.5, pp. 1857-1861, 2008.

16.G. Ghoniem, N. Faruqui, M. Elmissiry, A. Mahdy, H. Abdelwahab, M. Oommen et al. "Differential profile analysis of urinary cytokines in patients with overactive bladder". Intenational Urogynecology Journal. 2011;22, no. 8, 95361. vol. 22, pp. 953-61, 2011.

17.P. Tyagi, S. Motley, M. Kashyap, S. Pore, J Gingrich, Z. Wang et al. "Urine chemokines indicate pathogenic association of obesity with BPH/LUTS". International Urology and Nephrology. vol. 47, no. 7, pp. 1051-8, 2015. 